\title{
Correlation function analysis of fiber networks: implications for thermal conductivity
}

\author{
Jorge Martinez-Garcia, ${ }^{1}$ Leonid Braginsky, ${ }^{1,2}$ Valery Shklover, ${ }^{1}$ and John W. Lawson ${ }^{3}$ \\ ${ }^{1}$ Laboratory of Crystallography, Department of Materials, \\ ETH Zurich, 8093 Zurich, Switzerland \\ ${ }^{2}$ Institute of Semiconductor Physics, 630090 Novosibirsk, Russia \\ ${ }^{3}$ MS-234-1, NASA Ames Research Center, \\ Moffett Field, 94035 California, USA
}

\begin{abstract}
The heat transport in highly porous fiber structures is investigated. The fibers are supposed to be thin, but long, so that the number of the inter-fiber connections along each fiber is large. We show that the effective conductivity of such structures can be found from the correlation length of the two-point correlation function of the local conductivities. Estimation of the parameters, determining the conductivity, from the $2 \mathrm{D}$ images of the structures is analyzed.
\end{abstract}

PACS numbers: $65.80 .-\mathrm{g}, 44.30 .+\mathrm{v}, 68.65 .-\mathrm{k}, 44.35 .+\mathrm{c}$ 


\section{INTRODUCTION}

The composite materials comprising long fibers of high thermal conductivity immersed in some amorphous matrix of low thermal conductivity are now quite popular in applications. Such materials can be used as the thermal barrier coatings. The long fibers are necessary to ensure the mechanical strength of the coating. Long high-TC fibers are also useful to enable anisotropic properties of the coating. Ablative thermally protective systems is an example of such structures. These systems typically consist of low density but rigid carbon fiber material infiltrated with the phenolic resin. Such structures have a very low density ( $\sim 0.27 \mathrm{~g} / \mathrm{cm}^{3}$; this means that fibers occupy approx. $12 \%$ of the bulk of the specimen) ${ }^{1}$ the typical diameter of the carbon fibers is $14-16 \mu \mathrm{m}$ and their length exceeds $1600 \mu \mathrm{m}$. Using special manufacturing methods enables the parallel orientation of fibers in order to promote minimum through-the-thickness thermal conductivity, which is one of the critical properties.

In spite of many detailed studies, potential of highly-porous fiber materials seems to be not realized. Further progress depends on the possibility of accurate estimation and modeling of their properties. There exist some sophisticated approaches and software packages for modeling of these protection systems, for example the program package FIAT (Fully Implicit Ablation and Thermal response code) developed by NASA for one-dimensional (1D) simulation of kinetically controlled pyrolysis, in-depth conduction, blowing due to pyrolysis gases, surface recession as the function of time using 1D model of ablation of porous material. ${ }^{2-4}$ The program package TITAN, also developed by NASA, allows 2D modeling. ${ }^{5}$ Several other software packages for modeling of fiber materials properties are available: TITAN/GIANTS (integrated procedure), MARC (commercial FEM code) ${ }^{6}$, and data base TPSX (http://tpsx.arc.nasa.gov/). ${ }^{7}$

Nevertheless, direct numerical calculation of the transport properties in 3D fiber composites is very difficult (see, e.g., Ref. 8). Indeed, it is necessary to consider the 3D transport equation in a fine-structure mesh. The mesh spacing should be much smaller than the fiber thickness, while the total size of the mesh network should exceed the fiber length. Moreover, the composite structure is very complicated and its microscopic description requires a lot of parameters. ${ }^{9}$ The question arises whether all of these parameters are important for the thermal conductivity (TC) estimation or whether it can be estimated from a few other parameters related to them. We have shown that the two-point correlation function (CF) of 
the local conductivities is the only parameter, which is necessary for TC estimation in the non-homogeneous materials of low porosity. ${ }^{10,11}$ It is not clear, however, whether the CF is sufficient for the modeling of thermal conductivity of highly-porous fiber materials (porosity $\sim 80-90 \%$ ) or whether the higher order correlation functions should also be employed.

An important property of the fiber structures is their anisotropy. Anisotropy can be enabled artificially, however, a typical fiber structure is always anisotropic due to the nematic ordering. Effect of anisotropy on the electrical conductivity in such structures has been studied $^{12}$ and exact scaling laws have been obtained. ${ }^{13}$ The dependence of thermal conductivity of fiber composites on the fiber orientation was observed, e.g., in oriented fibrous carbon insulation. ${ }^{2}$ The room-temperature in-plane thermal conductivity of the carbon insulation at $1575 \mathrm{~K}$ was $0.05 \mathrm{~W} / \mathrm{mK}$ normal to the fibers plane and $0.14 \mathrm{~W} / \mathrm{mK}$ in the fibers plane. It was also reported, that the thermal conductivity of the fiber materials of the phenolic resin infiltration in the direction of fibers alignment is $\sim 2.4$ higher than that across the thickness. ${ }^{14}$ Also filled honeycombs, multilayers, tile-like structures are manufactured to provide anisotropic properties of the structure. ${ }^{15}$

Qualitative estimation of conductivity of the fiber structures has been done in Ref. 16 . It was shown that the conductivity can be expressed via porosity of the structure, thickness of the fibers, and mean distance between fiber connections. It is not clear, however, how to estimate these parameters from the two-point correlation function, which is not simple since CF cannot be approximated with a single exponent or Gaussian function. This problem will be considered in this paper. We show that total porosity and the correlation lengths along each $(x, y$, and $z)$ direction are the only parameters necessary for estimation of the conductivity of the fiber structures.

We start with a simple model of TC, which is a generalization of that proposed in Ref. 16 for an anisotropic media. We suppose the fibers are long enough, so that each of them has many crossing points along its length. This means also that the number of fibers per unit volume essentially exceeds the percolation threshold. This is the main point which distinguish our model from the previous studies where the conductivity near the percolation threshold has been investigated. ${ }^{12}$ We have found that TC can be expressed via porosity of the structure and mean distance between the fibers in each direction. In the following two sections we show that all these parameters can be estimated from the CF of the local conductivities. 
In Sec. III we consider the 2D models of the structures consisting of straight square fibers of equal thickness and length. By changing the length of the fibers, we can measure all parameters of the structure and compare them with the CF calculated using the image of the structure. We relate the parameters of the structure with the correlation lengths of $\mathrm{CF}$. We show that CFs of the structures with the same fiber thickness and main distance between the fibers are close. In Sec. IV we generalize this result to the 3D case.

\section{THERMAL CONDUCTIVITY OF FIBER STRUCTURES}

\section{A. Thermal conductivity of the Fiberform}

Fiberform is the structure composed exclusively of fibers without the resin infiltration. The heat propagation through the fiber network is the only mechanism of the thermal transport in such structure. By assuming the Fiberform as an array of cylindrically shaped fibers, each fiber can be geometrically characterized by its length $l$ and diameter $d$. If $n$ is the fiber concentration, i.e. the number of fibers in the unit cube, then these three parameters: $l, d$, and $n$ are the characteristic of the Fiberform. We introduce also the Fiberform density as $V_{f}=\pi n d^{2} l / 4\left(V_{f}=n d l\right.$ in the 2D case). All fibers are intersecting in a 2D model, if they are long enough and close. This is not the case in the 3D model, as not coplanar fibers can be skewed.

We suppose that the fibers are thin $(d \ll l)$ and long, so that the mean distance between the neighboring connecting points $\lambda$ is small compared to the fiber length, $(\lambda \ll l)$. This also means that the concentration of the fibers is large enough, so that we are well above the percolation threshold.

Suppose an external thermal gradient applied along the $z$-axis, and assume the cylindric symmetry of the Fiberform with regards to this axis, i.e., we consider the heat flow along the $z$-direction (Fig. 1). Let us choose two points in the fibers at the plane $z=$ const. The temperature, $T$, at these points can be different but its average values are equal. Indeed, the condition $\lambda \ll l$ means the existence of percolation between the points, while $\left\langle T\left(\boldsymbol{r}_{i}\right)\right\rangle \neq\left\langle T\left(\boldsymbol{r}_{k}\right)\right\rangle$ ensures the heat flux between them. The latter is forbidden by the cylindric symmetry, therefore $\left\langle T\left(\boldsymbol{r}_{i}\right)\right\rangle=\left\langle T\left(\boldsymbol{r}_{k}\right)\right\rangle$.

Let us find the heat flux, $I_{i j}$, through a fiber connecting two points $\boldsymbol{r}_{i}$ and $\boldsymbol{r}_{j}$ at two close 


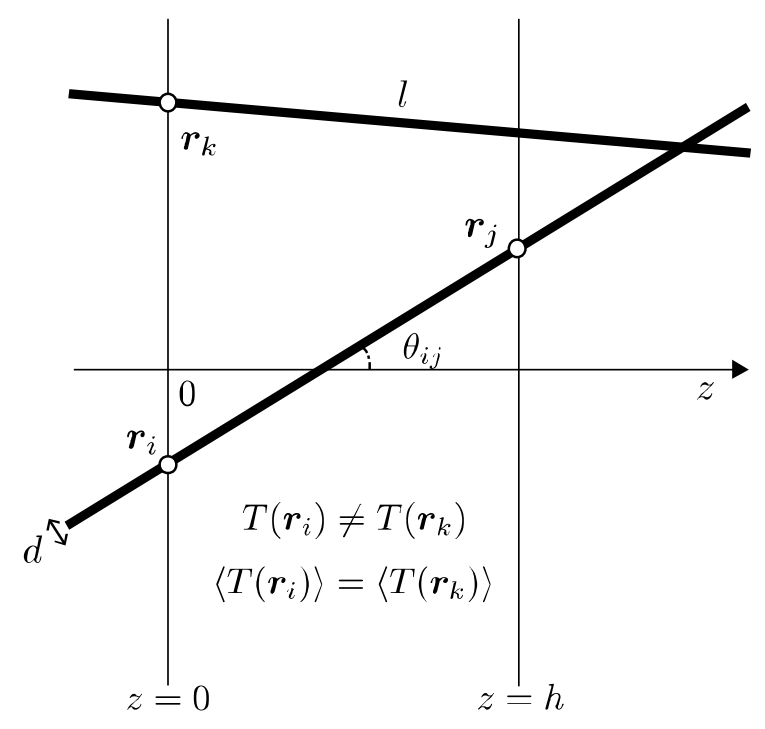

FIG. 1: Schematic of heat transport in a fiber structure. $z$ is the direction of heat flux.

planes $z=0$ and $z=h$, respectively (see Fig. 1 ):

$$
I_{i j}=\frac{\pi \kappa_{f} d^{2}}{4 l_{i j}}\left[T\left(\boldsymbol{r}_{i}\right)-T\left(\boldsymbol{r}_{j}\right)\right]
$$

Here $\kappa_{f}$ is the fiber thermal conductivity, $l_{i j}=\left|\boldsymbol{r}_{i}-\boldsymbol{r}_{j}\right|=h /\left|\cos \theta_{i j}\right|$, and $\theta_{i j}$ is the angle between the fiber and the $z$-axis. The total flux through the plane $z=0$ is equal to sum of all fluxes over all the fibers crossing the plane

$$
\Phi=\sum I_{i j}=\frac{\kappa_{\mathrm{eff}} S}{d}\left[\left\langle T\left(\boldsymbol{r}_{i}\right)\right\rangle-\left\langle T\left(\boldsymbol{r}_{j}\right)\right\rangle\right]
$$

This allows us to determine the effective thermal conductivity $\kappa_{\text {eff }}$ as:

$$
\kappa_{\mathrm{eff}}=\frac{\pi \kappa_{f} d^{2} N}{4 S\left[\left\langle T\left(\boldsymbol{r}_{i}\right)\right\rangle-\left\langle T\left(\boldsymbol{r}_{j}\right)\right\rangle\right]}\left\langle\cos \theta_{i j}\left[T\left(\boldsymbol{r}_{i}\right)-T\left(\boldsymbol{r}_{j}\right)\right]\right\rangle .
$$

Here $N=4 S / \pi D_{\perp}^{2}$ is the total number of fibers crossing the plane $z=0, S$ is the side area of the specimen in this plane, and $D_{\perp}$ is an average distance between the fibers crossing the plane $z=0$. Supposing that $\left\langle\cos \theta_{i j}\left[T\left(\boldsymbol{r}_{i}\right)-T\left(\boldsymbol{r}_{j}\right)\right]\right\rangle=\left\langle\cos \theta_{i j}\right\rangle\left\langle\left[T\left(\boldsymbol{r}_{i}\right)-T\left(\boldsymbol{r}_{j}\right)\right]\right\rangle$, one finds

$$
\kappa_{\mathrm{eff}}=\frac{d^{2}}{D_{\perp}^{2}} \kappa_{f} \quad \text { or } \quad \kappa_{\mathrm{eff}}=\frac{D_{\|}}{2 D_{\|}+D_{\perp}} p \kappa_{f}
$$

where $D_{\|}$is an average distance between the fibers along the $z$-direction, so that $V_{f}=$ $d^{2}\left(2 D_{\perp}+D_{\|}\right) /\left(D_{\perp}^{2} D_{\|}\right)$.

In a similar way we can consider the TC across the $z$-axis. The results are

$$
\kappa_{\|}=\frac{d^{2}}{D_{\perp}^{2}} \kappa_{f}, \quad \kappa_{\perp}=\frac{d^{2}}{D_{\|} D_{\perp}} \kappa_{f},
$$


or equivalently,

$$
\kappa_{\|}=\frac{D_{\|}}{2 D_{\|}+D_{\perp}} V_{f} \kappa_{f}, \quad \kappa_{\perp}=\frac{D_{\perp}}{2 D_{\|}+D_{\perp}} V_{f} \kappa_{f},
$$

where $\kappa_{\|}$and $\kappa_{\perp}$ are the components of the effective TC along and across the $z$-axis, respectively. Thus,

$$
\begin{aligned}
& \kappa_{\perp}+2 \kappa_{\|}=V_{f} \kappa_{f}, \\
& \kappa_{\perp} / \kappa_{\|}=D_{\perp} / D_{\|} .
\end{aligned}
$$

The former equality represents the trace of the conductivity tensor, which is invariant with respect to the axis used. The latter equality is in agreement with Ref. 13. In particular, for an isotropic structure $D_{\|}=D_{\perp}=D$ and the effective TC becomes

$$
\kappa_{\mathrm{eff}}=\frac{d^{2}}{D^{2}} \kappa_{f}=\frac{1}{3} V_{f} \kappa_{f} .
$$

The factor $1 / 3$ appearing in the last equation indicates that the rule of mixtures cannot be applied to the fiber structures. To understand the reason, let us consider the TC along the side of a simple cubic fiber network. We assume the thin fibers, so that the heat propagation exists only along the fibers, but does not across them. Therefore, only the fibers oriented along the applied temperature gradient participate in the TC. Effect of the other $2 / 3$ fibers can be estimated, if we replace the cube side $D$ by $D-d$. Then $\kappa_{\text {eff }}=d^{2} /(D-d)^{2} \approx$ $(1+2 d / D) d^{2} / D^{2}$. The factor $1 / 3$ disappears when $d \simeq D$.

\section{B. Thermal conductivity of ATPS}

ATPS is the material comprising the fibers immersed in some matrix of small TC, $\kappa_{M} \ll$ $\kappa_{f}$. The effect of the matrix can be two-fold. First, the direct heat flux across the matrix. Second, the heat transport through fibers and narrow bridges between two skewed fibers. The second effect can be important, if thermal resistivity of such bridges is of about the resistivity of the fibers between two connection points. Let us first consider the second effect.

Let $\delta$ be the length of such a bridge. The bridge is important if its resistivity is less or about the resistivity of the appropriate fiber part, i.e. $\delta / \kappa_{M} \lesssim \lambda^{*} / \kappa_{f}$. Here $\lambda^{*}$ is the

distance between two bridges which size is less than $\delta$. It can be estimated similar to that of Eq. (9):

$$
\lambda^{*}=\frac{3}{2 n(d+\delta / 2) l}
$$



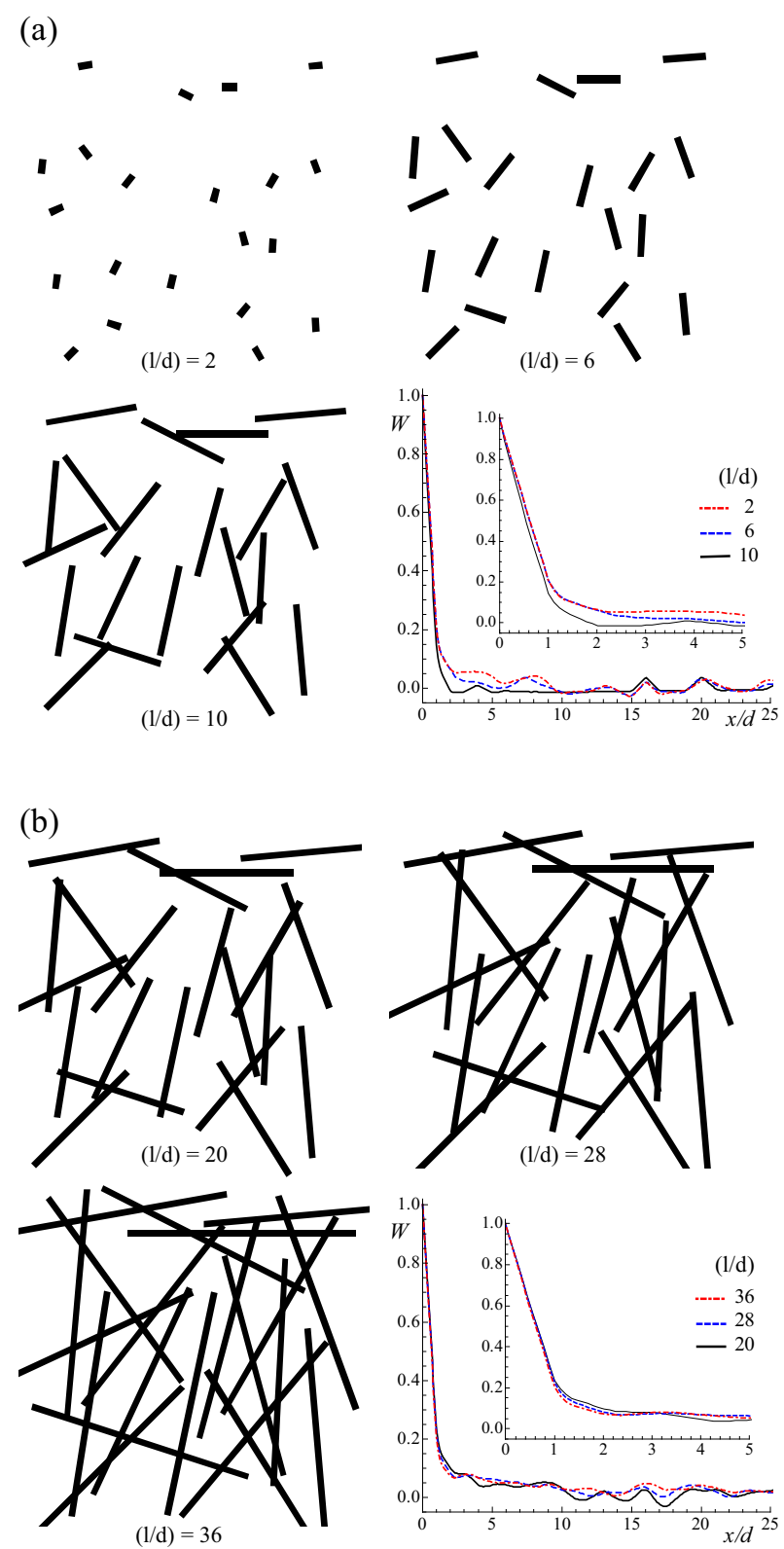

FIG. 2: Two dimensional models of fiber structures and corresponding correlation functions, $W$, taken along the horizontal axis, $x$. (a) Short-fiber models with aspect ratio, $(l / d)=2,6$ and 10 , $V_{f}=0.01,0.04$, and 0.07 , respectively. (b) Long-fiber models with aspect ratio, $(l / d)=10,20$ and $36, V_{f}=0.13,0.17$, and 0.21 , respectively. All fibers have the same positions, orientations and thickness in each model. A thickness value of $d=0.5$ unit was used in the computations.

Considering the cube, $\lambda^{*} \times \lambda^{*} \times \lambda^{*}$, we can write its resistivity as

$$
R=\left(\frac{\lambda^{*}}{\kappa_{f} d^{2}}+\frac{\delta}{\kappa_{M} d^{2}}\right) \frac{D^{2}}{\lambda^{*}}=\frac{1}{\lambda^{*} \kappa_{\mathrm{eff}}}
$$




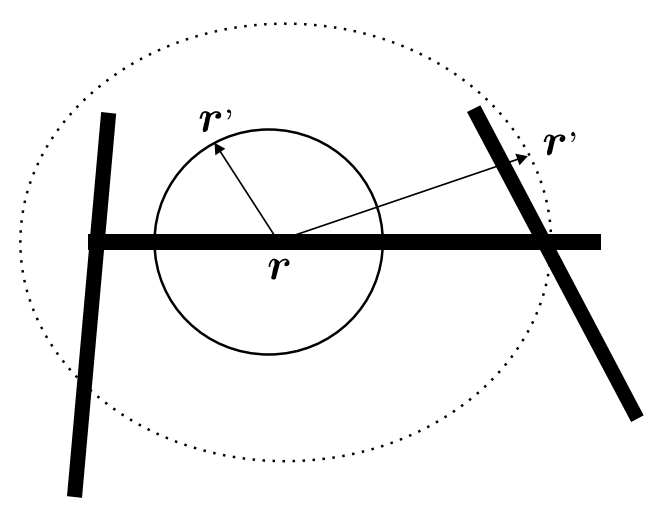

FIG. 3: Long fibers. Small circle intersects the fiber two times (bold line). Large circle has many points of intersection (dashed line).

from which one finds:

$$
\kappa_{\mathrm{eff}}=\frac{\lambda^{*} d^{2} / D^{2}}{\lambda^{*} / \kappa_{f}+\delta / \kappa_{M}}=\frac{d^{2} / D^{2}}{1 / \kappa_{f}+\delta / \lambda^{*} \kappa_{M}} .
$$

The above relation coincides with a similar estimation for $\kappa_{\text {eff }}$ in Ref. 16, if we suppose, $\delta / \lambda^{*}=d^{2} / \lambda^{2}$ or $\delta \simeq V_{f} d$. Equation (4) turns into Eq. (1), if the last term in its denominator is omitted. This is possible when $\delta / \lambda^{*} \ll \kappa_{M} / \kappa_{f}$ or $V_{f} \delta^{2} / d^{2} \ll \kappa_{M} / \kappa_{f}$.

Another way to take the bridges into account consists in assuming each fiber surrounded by some layer, so that the thickness of each fiber is $\delta_{0}>d$ and its effective TC is, $\tilde{\kappa}=$ $\left[\kappa_{f} d^{2}+\kappa_{M}\left(\delta_{0}^{2}-d^{2}\right)\right] / \delta_{0}^{2}$. Substituting the latter into Eq. (1) with $d \rightarrow \delta_{0}$ yields:

$$
\kappa_{\mathrm{eff}}=\frac{\kappa_{f} d^{2}+\kappa_{M}\left(\delta_{0}^{2}-d^{2}\right)}{D^{2}}
$$

This means that the fiber network and the matrix can be considered as two resistors connected in parallel. Therefore, the TC of the structure can be estimated as: ${ }^{17}$

$$
\kappa_{\mathrm{eff}}=\frac{1}{3} V_{f} \kappa_{f}+\left(1-\frac{4}{3} V_{f}\right) \kappa_{M}
$$

\section{CORRELATION FUNCTION OF THE 2D FIBER STRUCTURES}

In order to estimate the parameters $D_{\|}$and $D_{\perp}$ required for the TC modeling, we consider the CF of fiber structures. We introduce CF as

$$
W\left(\boldsymbol{r}, \boldsymbol{r}^{\prime}\right)=\frac{\left\langle[\eta(\boldsymbol{r})-\langle\eta(\boldsymbol{r})\rangle]\left[\eta\left(\boldsymbol{r}^{\prime}\right)-\left\langle\eta\left(r^{\prime}\right)\right\rangle\right]\right\rangle}{4 V_{f}\left(1-V_{f}\right)},
$$


where $\langle\cdots\rangle$ denotes an ensemble average and the characteristic function $\eta(\boldsymbol{r})$ is given by

$$
\eta(\boldsymbol{r})=\left\{\begin{aligned}
1, & \text { if } \boldsymbol{r} \text { is inside a fiber } \\
-1, & \text { otherwise }
\end{aligned}\right.
$$

For the statistically homogeneous media, Eq. (6) depends only on the difference between two arbitrary points $\boldsymbol{r}$ and $\boldsymbol{r}^{\prime},\left[W\left(\boldsymbol{r}, \boldsymbol{r}^{\prime}\right)=W\left(\boldsymbol{r}-\boldsymbol{r}^{\prime}\right)\right]$; it is equal to unity at the coordinates origin, $W(0)=1$ and vanishes at the infinity. If the media is also isotropic, then $W\left(\boldsymbol{r}, \boldsymbol{r}^{\prime}\right)=$ $W\left(\left|\boldsymbol{r}-\boldsymbol{r}^{\prime}\right|\right)$. The aim of this section is to calculate the CF using the structure image and estimate the parameters of the structure from the appropriate correlation lengths. We will see that the CF is determined by the fiber thickness $d$ and the distance between the intercrossing points of the fibers, if their density is large enough. Otherwise, the fiber length becomes the second correlation length. In evaluating $\mathrm{CF}$ from digital images we employed the procedure outlined in Ref. 18.

Figure 2 displays the 2D fiber models and the corresponding CF. All the structures are composed of similar fibers; they have the same positions and orientations, and can be distinguished by the fiber length only. Fibers in Figure 2(a) are short, so that they do not intersect. The corresponding $\mathrm{CF}$ have two correlation lengths: one of them is relevant to the fiber thickness $(r / d=1)$ and the other one to the fiber length. The CF of different structures of this figure are well distinguished.

On the contrary, the CF in Fig. 2(b) are very close. This is because the second correlation length for the long fibers is the mean distance between the inter-crossing points, but not the fiber length. Direct calculation of this distance (from the image using, e.g., Photoshop softwear) yields about $9 d$ for all the structures in Fig. 2(b).

In order to understand the difference between the CF behavior in Fig. 2(a) and Fig. 2(b), let us consider the two-point probability function ${ }^{19,20}$

$$
S=\left\langle\eta_{0}(\boldsymbol{r}) \eta_{0}\left(\boldsymbol{r}^{\prime}\right)\right\rangle
$$

where

$$
\eta_{0}(\boldsymbol{r})=\left\{\begin{array}{l}
1, \text { if } \boldsymbol{r} \text { is inside a fiber } \\
0, \text { otherwise. }
\end{array}\right.
$$

Then $S=V_{f}$, if $\left|\boldsymbol{r}-\boldsymbol{r}^{\prime}\right| \ll d$, i.e. if both $\boldsymbol{r}$ and $\boldsymbol{r}^{\prime}$ are either inside or outside the fiber. In order to estimate $S$ for a longer distance, $\left|\boldsymbol{r}-\boldsymbol{r}^{\prime}\right| \gg d$, we have to multiply the 
(a)
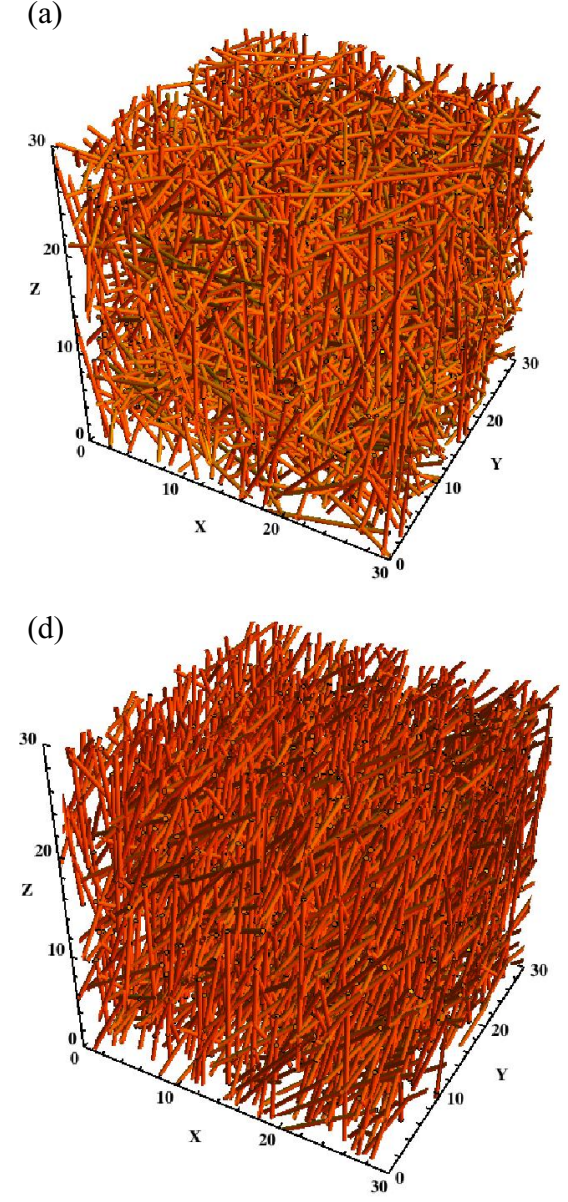

(b)

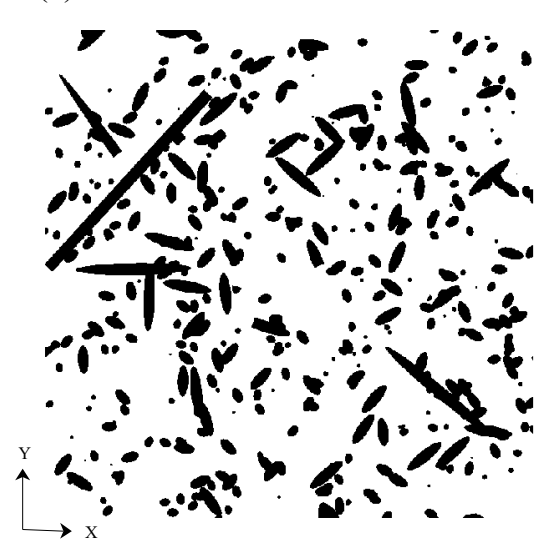

(e)

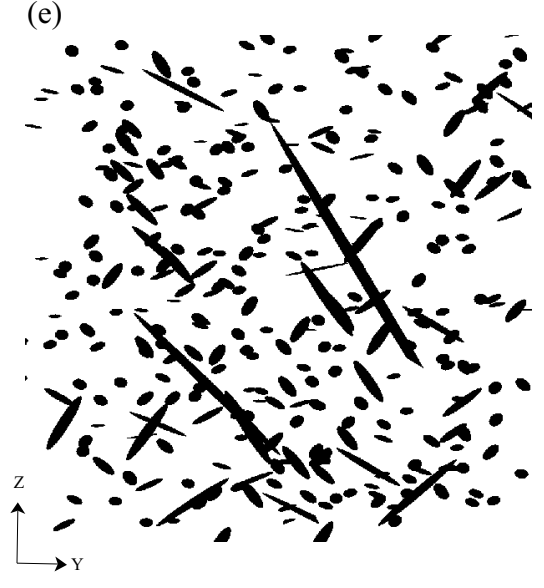

(c)

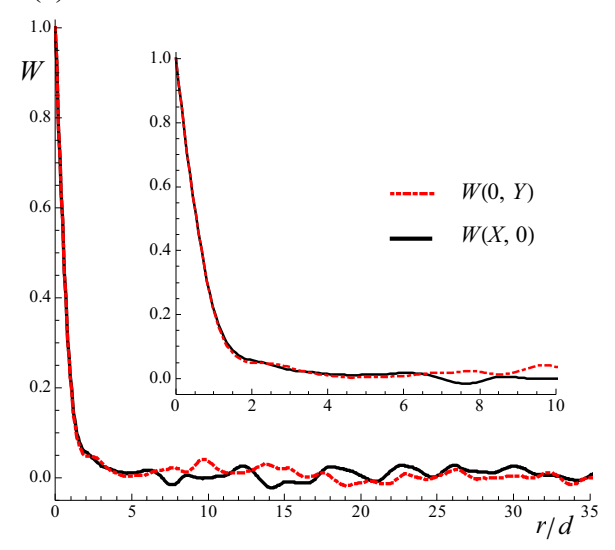

(f)

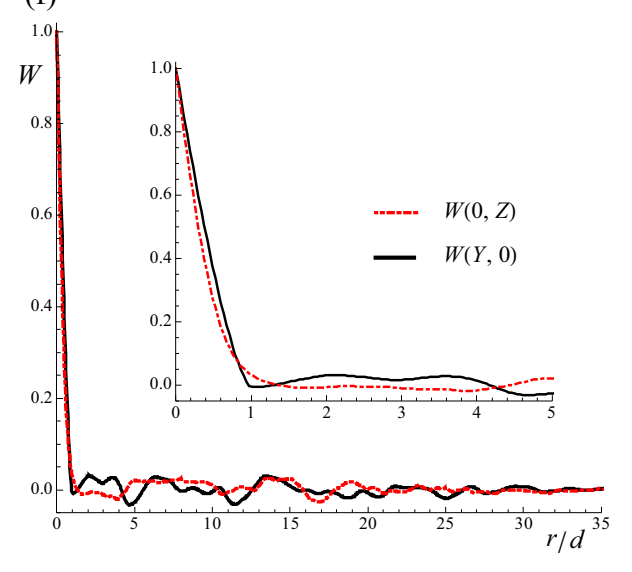

FIG. 4: Cross-sections images and corresponding correlation functions of 3D models of fiber structures. (a) Fibers randomly positioned and oriented in the space. (b) Cross section image of (a) at $z=15$. (c) Correlation functions $W(x, 0)$ and $W(0, y)$ of (b), taken along the $x$ and $y$ axis respectively. (d) Fibers randomly positioned with preferred orientation along the $z$-axis. (e) Cross section image of (d) at $x=10$. (f) Correlation functions of (e) taken along the $y$ and $z$ axis. A total number of $N=2000$ cylindrical shaped fibers with aspect ratio $(l / d)=40$ and $V_{f}=0.21$, were considered in the simulations.

probability that the point $\boldsymbol{r}$ belongs to the fiber $\left[P(\boldsymbol{r} \in\right.$ fiber $\left.)=V_{f}\right]$ by the probability for the point $\boldsymbol{r}^{\prime}$ belongs to the fiber $\left[P\left(\boldsymbol{r}^{\prime} \in\right.\right.$ fiber $\left.)\right]$ provided by the first point $\boldsymbol{r}$ is in the fiber. The latter probability can be easily estimated, if we suppose isotropy of the fiber structure and draw a circle around $\boldsymbol{r}$, as shown in Figure 3. Then $P\left(\boldsymbol{r}^{\prime} \in\right.$ fiber $)$ can be estimated as the ratio of the fiber thickness inside the circle to the circle length. For the short fibers [Fig. 2(a)], $P\left(\boldsymbol{r}^{\prime} \in\right.$ fiber $)=d / \pi\left|\boldsymbol{r}-\boldsymbol{r}^{\prime}\right|$, if $d \ll\left|\boldsymbol{r}-\boldsymbol{r}^{\prime}\right| \ll l$, and $P\left(\boldsymbol{r}^{\prime} \in\right.$ fiber $)=V_{f}$, 
if $\left|\boldsymbol{r}-\boldsymbol{r}^{\prime}\right| \gg l$; in the latter case both probabilities are independent. For the long fibers [Fig. 2(b)], $P\left(\boldsymbol{r}^{\prime} \in\right.$ fiber $)=d / \pi\left|\boldsymbol{r}-\boldsymbol{r}^{\prime}\right|$, if $d \ll\left|\boldsymbol{r}-\boldsymbol{r}^{\prime}\right| \ll l_{c}$, where $l_{c}$ is the mean distance between the crossing points. Note that $P\left(\boldsymbol{r}^{\prime} \in\right.$ fiber $)$ has an increase, when $\left|\boldsymbol{r}-\boldsymbol{r}^{\prime}\right|>l_{c}$. Such increase can be found in the CF of Fig. 2(b) at $\left|\boldsymbol{r}-\boldsymbol{r}^{\prime}\right| \approx 10 d$. For $\left|\boldsymbol{r}-\boldsymbol{r}^{\prime}\right| \gg l_{c}$, $S=V_{f}^{2}$ as $P(\boldsymbol{r} \in$ fiber $)$ and $P\left(\boldsymbol{r}^{\prime} \in\right.$ fiber $)$ are independent at the large distances. From the above, Eq. (7) can be rewritten as

$$
S= \begin{cases}V_{f}, & \left|\boldsymbol{r}-\boldsymbol{r}^{\prime}\right| \ll d, \\ \frac{d}{\pi\left|\boldsymbol{r}-\boldsymbol{r}^{\prime}\right|}, & d \ll\left|\boldsymbol{r}-\boldsymbol{r}^{\prime}\right| \ll l_{c}, \\ V_{f}^{2}, & \left|\boldsymbol{r}-\boldsymbol{r}^{\prime}\right| \gg l_{c} .\end{cases}
$$

Since $\eta(r)=2 \eta_{0}(r)-1$, it follows from Eqs. (6) and (7) that

$$
W\left(\boldsymbol{r}-\boldsymbol{r}^{\prime}\right)=\frac{S\left(\boldsymbol{r}-\boldsymbol{r}^{\prime}\right)-V_{f}^{2}}{V_{f}\left(1-V_{f}\right)},
$$

thus

$$
W\left(\boldsymbol{r}-\boldsymbol{r}^{\prime}\right)= \begin{cases}1, & \left|\boldsymbol{r}-\boldsymbol{r}^{\prime}\right| \ll d, \\ \frac{d}{\pi\left|\boldsymbol{r}-\boldsymbol{r}^{\prime}\right|\left(1-V_{f}\right)}-\frac{V_{f}}{1-V_{f}}, & d \ll\left|\boldsymbol{r}-\boldsymbol{r}^{\prime}\right| \ll l_{c}, \\ 0, & \left|\boldsymbol{r}-\boldsymbol{r}^{\prime}\right| \gg l_{c} .\end{cases}
$$

A similar behavior of CF was found in Fig. 2(b). Note that $d /\left|\boldsymbol{r}-\boldsymbol{r}^{\prime}\right| \gg V_{f}$, if $\left|\boldsymbol{r}-\boldsymbol{r}^{\prime}\right| \ll l_{c}$.

Apparently, it is the connectivity $(\lambda)$, i.e. the mean distance between the crossing points, but not the mean length of the fibers that determines the transport properties in the fiber structure. We have shown that the connectivity coincides with the correlation length of the $\mathrm{CF}$ in the $2 \mathrm{D}$ case, $\lambda \simeq l_{c}$. Therefore it can be estimated from Eq. (6). It is the connectivity which determines the conduction pathways. They are roughly the same in the Fig. 2(b) thus,

all these structures have the same TC. Elongation of the fibers in this case means increase of the dead ends, but it does not change the conductivity. Increase of the TC can be expected when the fibers becomes so long that the number of the crossing points increases. This means decrease the connectivity value and, therefore, increase of the conductivity.

\section{CORRELATION FUNCTION OF THE 3D FIBER STRUCTURES}

The main difference between the 2D and 3D models concerns the intersection of the fibers. Indeed, the fibers in the 2D model intersect, if they are long enough and close. In 
general, the fibers in the 3D structure can be skewed; this happens with the fibers which are not coplanar. If so, then the mean distance between the crossing points should exceed the distance between the fibers. The question arises what is the second correlation length discussed in the previous section? Is it relevant to the connectivity or to the mean distance between the fibers?

Let us first estimate the connectivity $\lambda$. Consider a large parallelepiped, $L \times L \times \lambda$ $(L \rightarrow \infty)$. The number of fibers within it is $N=n L^{2} \lambda$, and their total projection on the $L \times L$ plane is, $(2 / 3 d l) N$. This projection should cover the entire plane, if $\lambda$ is the distance between the crossing points. Thus,

$$
\lambda=\frac{3}{2 n d l} .
$$

The mean distance between the fibers $D$, can be estimated as $D^{2} n l=1$, or

$$
D=\frac{1}{\sqrt{n l}} .
$$

Then for the ratio $\lambda / D$, we obtain

$$
\frac{\lambda}{D}=\frac{3}{4} \sqrt{\frac{\pi}{V_{f}}} \approx \frac{1}{\sqrt{V_{f}}},
$$

where $V_{f}=\pi n d^{2} l / 4$ is the density of the $3 \mathrm{D}$ fiber structure. This value is not so large for $V_{f} \simeq 0.1$.

It is interesting to compare $\lambda$ with the fiber length $l$ :

$$
\frac{\lambda}{l}=\frac{3}{2 n d l^{2}} .
$$

From this relation it is evident that $\lambda \sim l$, if $n d l^{2} \sim 1$, i.e., the fiber concentration $n_{c}=\left(d l^{2}\right)^{-1}$ determines the percolation threshold. Indeed, at $n=n_{c}$ we have only one fiber in the $l \times l \times d$ box. The fibers are not connected, if $n \ll n_{c}$, but connected in the opposite limit. The mean number of connections per fiber is, $l / D \sim l \sqrt{l n}$. In this paper we assume this value to be large.

For a rough estimation of the correlation function in 3D we have to replace the circle in Fig. 3 with a sphere, so that the conditional probability $P\left(\boldsymbol{r}^{\prime} \in\right.$ fiber $)$ provided by the first point $\boldsymbol{r}$ is in the fiber is now, $P\left(\boldsymbol{r}^{\prime} \in\right.$ fiber $)=d^{2} / 8\left|\boldsymbol{r}-\boldsymbol{r}^{\prime}\right|^{2}$. Therefore Eq. (8) becomes:

$$
W\left(\boldsymbol{r}-\boldsymbol{r}^{\prime}\right)= \begin{cases}1, & \left|\boldsymbol{r}-\boldsymbol{r}^{\prime}\right| \ll d, \\ \frac{d^{2}}{8\left|\boldsymbol{r}-\boldsymbol{r}^{\prime}\right|^{2}\left(1-V_{f}\right)}-\frac{V_{f}}{1-V_{f}}, & d \ll\left|\boldsymbol{r}-\boldsymbol{r}^{\prime}\right| \ll D, \\ 0, & \left|\boldsymbol{r}-\boldsymbol{r}^{\prime}\right| \gg D .\end{cases}
$$




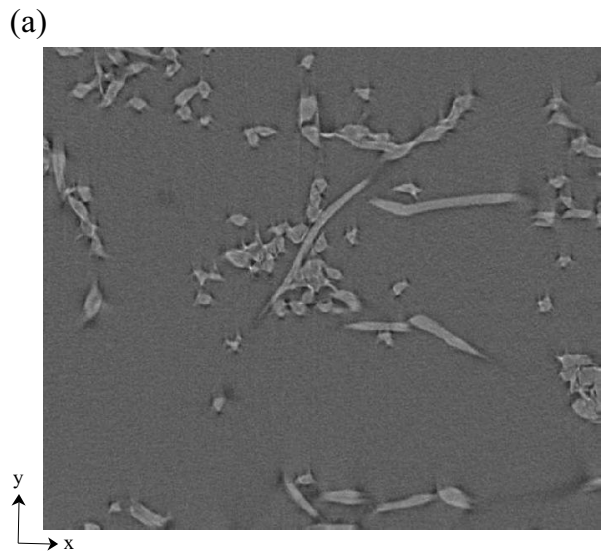

(b)

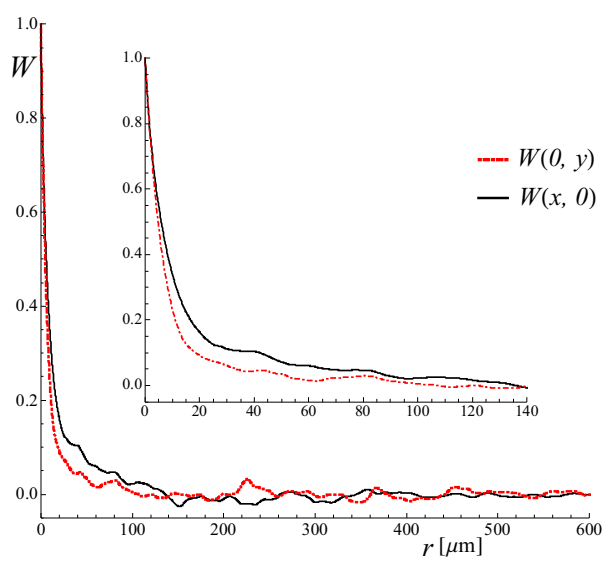

(c)

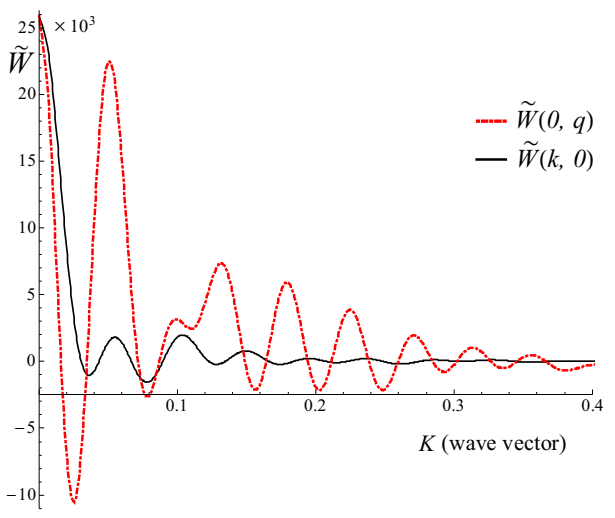

FIG. 5: Cross-section of a 3D Fiberform (a), its correlation function, $W$ (b), and Fourier transform of $W$ in $x$ and $y$ directions. [Image (a) provided to NASA courtesy of Xradia, Inc.]

For the correlation length we obtain thus, $l_{c} \simeq D$.

Just with the purpose to illustrate how the 3D case can be treated, we have simulated 3D fiber structures and extracted the cross-section images from them (Fig. 4). The algorithm employed to produce such structures allows free intercrossing of the fibers, so that nematic 
ordering does not occur. It relies on the basic ideas exposed by Berryman, ${ }^{18}$ but it has been readapted to the cylindrical geometry in this work. Figures 4(b) and 4(c) displays one of such cross-sections $(z=15)$ and the corresponding $W(x, 0), W(0, y)$ of a fully random fiber structure. As it can be appreciated, these functions are very close because both $x$ and $y$ direction are equal. The CF allows to estimate the mean distance among fibers as $D=2 d$. This does not occur when the fibers have a preferred orientation [Fig. 4(d)] and we examinate a cross-section containing the preferred axis [Fig. 4(e)]. The CF measured along the preferred axis and along the direction perpendicular to it, display different correlation lengths as shown in Figure 4(f).

It is interesting to consider the Fourier transform of the correlation function. For the simple CF given in Eq. (10) and $d \ll D$, it has the form

$$
\tilde{W}(k)=\frac{4 \pi V_{f}}{\left(1-V_{f}\right) k^{3}}(\sin k D-k D \cos k D) .
$$

In Eq. 11 we omit the small values, which oscillate as $\cos k d$. Thus, we can estimate the $D$ value analyzing the oscillations of Fourier transform of the CF. This is important for the correlation functions measured from the structure images.

Figure 5 illustrates the cross-section of a 3D Fiberform material, its correlation function and the Fourier transform. It is not easy to estimate the correlation length directly from the CF, because of the noise [Fig. 5(b)]. However, it can be estimated from the Fourier transform: the period of the shortest oscillations is equal to $2 \pi / D$. For an anisotropic structure, as that shown in Fig. 5(a), this criteria allows estimation of the ratio $D_{\perp} / D_{\|}$, which determines the anisotropy of the thermal conductivity along and across the structure axis.

Let us estimate the thermal conductivity of the Fiberform presented in Fig. 5. Volume fraction of the fibers can be calculated directly from the digital image of Fig. 5(a), we found it equal to $V_{f}=0.1$. Diameter of the fibers is the first correlation length of CF; we found $d \approx 15 \mu \mathrm{m}$ from Fig. $5(\mathrm{~b})$. If we assume for the fiber length $l=1600 \mu m$, then for the fiber concentration, $n=4 V_{f} / \pi d^{2} l$, we found $n \approx 3.5 \cdot 10^{5} \mathrm{~cm}^{-3}$; this value much exceeds the percolation threshold $n_{c}=1 / \mathrm{dl}^{2} \approx 2.6 \cdot 10^{4} \mathrm{~cm}^{-3}$. Rough estimation of the mean distance between the fiber connections is, $\lambda=3 / 2 n d l \approx 180 \mu m$; so that there are about $l / \lambda \approx 10$ connections for each fiber. Thus, all assumptions previously made to estimate the heat transport are satisfied. The distances between two adjacent peaks in both, bold and dashed, 
curves in Fig. 5(c) are equal to $0.045 \mu \mathrm{m}^{-1}$. This means that $D_{\|}=D_{\perp} \approx 140 \mu \mathrm{m}$. This estimate can be observed in Fig. $5(\mathrm{~b})$ as well. Then Eq. (3) yields $\kappa_{\|}=\kappa_{\perp} \approx 0.03 \kappa_{f}$.

\section{DISCUSSION}

We found the inequality $n \gg n_{c}$ holds well for the structure Fig. 5. The same estimation can be done also for the ablative thermal protection structure ${ }^{1}$ mentioned in the Introduction. Note also that of the percolation threshold $V_{f c}=\pi d^{2} l n_{c} / 4$, which has been estimated in Ref. 12 for $l / d=80$, yields $V_{f c}=0.03$, while typical volume concentration of the fibers in such structures is $V_{f}=0.1-0.2$. This means that Eq. (3) can be used for the TC estimation, and the result is in agreement with the experiments. ${ }^{2,14}$

More interesting is the effect of anisotropy. In Sec. II we found the simple estimation Eq. (2), which depends on the correlation lengths $D_{\|}$and $D_{\perp}$, but is independent on the connectivity $\lambda$. This result seems surprising, but it can be understood from the following example. Let us consider the thermal conductivity of a cubic mesh of fibers. It can be estimated as the thermal conductivity of the $\lambda \times \lambda \times \lambda$ cube. The number of disconnected fibers within the cube is $(\lambda / D)^{2}$ and the resistivity of each fiber is, $4 \lambda / \pi d^{2} \kappa_{f}$. The resistivity of the cube can then be written as $\left(4 \lambda / \pi d^{2} \kappa_{f}\right) \cdot\left(\pi D^{2} / 4 \lambda^{2}\right)$ or $1 / \lambda \kappa_{\text {eff }}$. Thus,

$$
\kappa_{\mathrm{eff}}=\left(\frac{d}{D}\right)^{2} \kappa_{f} .
$$

This result would not changed, if we had disconnected some of the contacts in the cubic mesh. The result changes only if we disconnect all the contacts along the fibers. Therefore, the error of the estimation Eq. (3) cannot be better than $\lambda / l \sim n_{c} / n$. This is the effect of dead ends, which is not important far from the percolation threshold $n_{c}$. For the same reason, the small bridges of the matrix material do not affect the thermal conductivity in the case of the immersed fibers.

Another reason for the error in Eq. (3) comes from the dispersion of resistivity of the fiber segments between two contacts. This effect can be estimated with the factor ${ }^{21}$

$$
\Phi=\left(1+\frac{2 \sigma^{2}}{N}\right)^{-1}
$$

where $\sigma$ is the relative dispersion of the resistivity (i.e., relative dispersion of the length between adjacent contacts in our case) and $N \geq 6$ in the 3D resistor net. Assuming $\sigma \lesssim 0.5$, we estimate the relative error as 0.1 . 
Typically, the matrix material (phenolic resin) can be porous itself. The size of its pores is very small, and their effect can be estimated, if we make the substitution ${ }^{17} \kappa_{M} \rightarrow$ $\kappa_{M}\left(1-\frac{4}{3} p_{M}\right)$, where $p_{M}$ is the intrinsic matrix porosity. This makes the effect of the matrix inessential.

Estimation of the conductivity of anisotropic structures can be performed using Eq. (3). Indeed, the fiber density $V_{f}$ can be obtained from the structure porosity, while the ratio of the correlation lengths, $D_{\perp} / D_{\|}$, can be estimated by analyzing the oscillations of the Fourier transforms of the CF across and along the structure [Fig. 5(b)].

In order to calculate the correlation function, we have used the model structures. The same calculation can be made also using the real SEM or optical images of the cross-sections. Similar images can be obtained from the X-ray tomography [Fig. 5(a)]. Basically one slice is enough for the CF calculation; however its accuracy can be significantly increased, if we use a few slices and average the results of calculations.

In conclusion, we have analyzed the expressions (2) for the thermal conductivity of the fiber structures and investigated the estimation of their parameters from the information provided by the $\mathrm{CF}$.

This work was supported by the Swiss National Science Foundation (grant 200021 $130274 / 1)$.

1 M. Stackpole, S. Sepka, I. Cozmuta and D. Kontinos. Post-flight evaluation of Stardust sample return capsule forebody heatshield material. AIAA 2008-1202, 46th AIAA Aerospace Sciences Meeting, January 2008.

2 T. G. Godfrey, D. L. McElroy, Z. L. Ardary, Nucl. Technol. 22, 94 (1974).

3 Y. -K. Chen, Frank S. Milos, J. Spacecraft and Rockets, 36, 475 (1999).

4 M. A. Covington, J. M. Heinemann, H. E. Goldstein, Y. -K. Chen, I. Terrazas-Salinas, J. A. Balboni, J. Olejniczak and E. R. Martinez. Performance of a low-density ablative heat shield material. AIAA Paper 2004-2273, June 28, 2004.

5 Y. -K. Chen and W. D. Henline. Analysis of Hypersonic Arcjet Flowfields and Surface Heating of Blunt Bodies, AIAA Paper 93-027, January 1993.

6 Users Manual, MARC Analysis Research Corporation, Volume A: Users Information, MARC 
Analysis Research Corp., Palo Alto, CA, p. E5.0.1-A1.8, 1994

7 T. H. Squire, F. S. Milos, G. C. Hartlieb and D. J. Rasky. TPSX: Thermal Protection System Expert and Material Property Database," ICCE/4, Fourth International Conference on Composite Engineering, Ed. D. Hui, International Community for Composites Engineering and College of Engineering, Univ. of New Orleans, LA, p. 937, 1997.

8 O. Coindreau and G. L. Vignoles, J. Mater. Res., 20, 2328 (2005).

9 G. L. Vignoles, O. Coindreau, A. Ahamadi and D. Bernard, J. Mater. Res., 22, 1537 (2007).

10 L. Braginsky, V. Shklover, G. Witz and H.-P. Bossmann, Phys. Rev. B, 75, 094301 (2007).

11 L. Braginsky and V. Shklover, Phys. Rev. B, 78, 224205 (2008).

12 S. White, B. DiDonna, M. Mu, T. Lubensky, and K. Winey, Phys. Rev. B, 79, 024301 (2009).

13 X. Zheng, M. G. Forest, R. Lipton, R. Zhou and Q. Wang, Adv. Funct. Mater. 15, 627 (2005).

14 M. Moniruzzamann and K. I. Winey, Macromolecules, 39, 5194 (2006).

15 F. S. Milos, Y. -K. Chen. Two-dimensional ablation, thermal response, and sizing program for pyrolizing ablators. AAIA Paper 2008-1223, Jan. 2008.

16 T. Hu, A. Yu. Grosberg and B. I. Shklovskii, Phys. Rev. B, 73, 155434 (2006).

17 P. G. Klemens, High Temp. - High Press. 23, 241 (1991).

18 J. G. Berryman, J. Appl. Phys. 57, 2374 (1985).

19 S. Torquato, Random Heterogeneous Materials: Microstructure and Macroscopic properties, (Springer-Verlag, New York, 2002).

20 M. Sahimi, Heterogeneous Materials I. Linear Transport and Optical Properties, (SpringerVerlag, New York, 2003).

21 L. Braginsky, L. Lukzen, V. Shklover, and H. Hofmann, Phys. Rev. B, 66, 134203 (2002). 